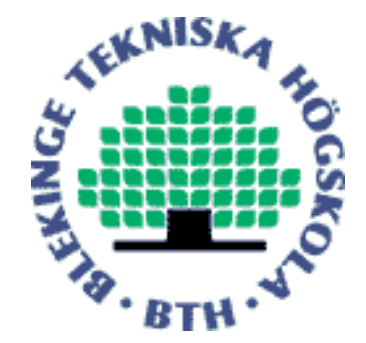

Copyright (C) 2012 IEEE.

Citation for the published paper:

Adapting the Streaming Video Based on the Estimated Position of the Region of Interest

Hussein Muzahim Aziz, Markus Fiedler, Håkan Grahn, Lars Lundberg

Eighth International Conference on Signal Image Technology and Internet Based Systems (SITIS)

2012 Naples

This material is posted here with permission of the IEEE. Such permission of the IEEE does not in any way imply IEEE endorsement of any of BTH's products or services Internal or personal use of this material is permitted. However, permission to reprint/republish this material for advertising or promotional purposes or for creating new collective works for resale or redistribution must be obtained from the IEEE by sending a blank email message to pubs-permissions@ieee.org.

By choosing to view this document, you agree to all provisions of the copyright laws protecting it. 


\section{Adapting the Streaming Video Based on the Estimated Position of the Region of Interest}

\author{
Hussein Muzahim Aziz \\ School of Computing \\ Blekinge Institute of \\ Technology, \\ 37179 Karlskrona, Sweden \\ $+46455385876$ \\ hussein.aziz@bth.se
}

\author{
Markus Fiedler \\ School of Computing \\ Blekinge Institute of \\ Technology, \\ 37179 Karlskrona, Sweden \\ $+46455385653$ \\ markus.fiedler@bth.se
}

\author{
Håkan Grahn \\ School of Computing \\ Blekinge Institute of \\ Technology, \\ 37179 Karlskrona, Sweden \\ $+46455385804$ \\ hakan.grahn@bth.se
}

\author{
Lars Lundberg \\ School of Computing \\ Blekinge Institute of \\ Technology, \\ 37179 Karlskrona, Sweden \\ $+46455385833$ \\ lars.lundberg@bth.se
}

\begin{abstract}
Streaming real time video over wireless networks cannot guarantee that all the frames could meet their deadlines. Wireless networks may suffer from bandwidth limitations. To reduce the streaming data over wireless networks, we propose a technique to identify, and extract the Region Of Interest (ROI), and drop the non-ROI from the frames that are between the reference frames. The Sum of Absolute Differences (SAD) will compute the consecutive video frames to identify the ROI as it considered the most motion and important region. The reconstruction mechanism to the non-ROI is performed on the mobile side by applying linear interpolation between the reference frames. We evaluate the proposed approach by using Mean Opinion Score (MOS) measurements. MOS are used to evaluate the two scenarios with equivalent encoding size, where the users observe the first scenario with a low bit rate for the original videos, while for the second scenario the users observe our proposed approach. The results show that our technique significantly reduces the amount of data, while the reconstruction mechanism provides acceptable video quality to the mobile viewers.
\end{abstract}

Keywords - streaming video; region of interest; sum of absolute differences; mean opinion scores

\section{INTRODUCTION}

Real time video transmission over wireless networks faces several challenges such as high error rate, bandwidth variations and limitations, and the capability constraints on the mobile devices. Among these, the bandwidth variations and limitations of the wireless channel that considered as the major challenge for video streaming [1]. Streaming high amount of video data could increase the network traffics. Therefore, it is desirable to adjust the transmission rate according to the perceived congestion level to maintain a suitable losses level in wireless networks.

Bandwidth considered as one of the most critical resources in wireless networks, as the available bandwidth should be managed efficiently [2]. Therefore, the data size of a video stream should be adapted according to the network bandwidth [3],[4].

Network adaptation refers to how much network resources (e.g., bandwidth) a video stream should be utilize for video content, resulting in designing an adaptive streaming mechanism for video transmission [5].
The main feature of H.264/SVC [6] is to provide bandwidth-optimized transmission for video streaming by observing current network conditions. H.264/SVC provides three types of enhancements for optimized bandwidth transmission. First, it can support spatial enhancements of quality through a signal-noise-ratio. Second, it can support temporal enhancements by changing the frame rate, and finally it can support spatial enhancements through resolution.

The basic element of H.264/AVC video sequence is slicing, where each frame are divided into several slices [7] and each slice contains a group of macroblocks (MBs) [8], [9]. H.264/AVC introduces flexible macroblock ordering (FMO) as a useful error resilient tools, where H.264/AVC defines seven different types of FMO modes: Interleaved, Dispersed, Fore-ground with left-over, Box-out, Rasterscan, Wipe, and Explicit [10].

The H.264/SVC encodes the video in the way that can be selectively transmitted according to the type option; contents and network condition by using a bit stream extractor [11].

The user attention is the ability to identify the interested parts for a given scene, called attention area or Region Of Interest (ROI). The ROI could be extracted from the streaming video, as it consider the most motion, interesting and important parts in the video frame, while the non-ROI (background) is considered less important region [12].

H.264 contains a rate-control algorithm that are dynamically adjusts the encoder parameters to achieve a target bit rate by allocates a budget of bits to the video frames sequence. The main concept of the rate-control algorithm is a quantitative model, which describe the relationship between the quantization parameter (QP) and the actual bit rate [13]. QP has a great impact on the encoder performance, because it regulates how much spatial details can be saved. As the increases of the QP, some of the details are aggregated so that the bit rates drops with some increases in distortion and some losses of the video quality [14].

In this paper, we propose a technique to identify and extract the ROI between the reference frames on the server side. Extracting the ROI and drop the non-ROI is to reduce the amount of data to be streamed over wireless networks. 
The reference frames and the ROI will be streamed to the mobile device. Linear interpolation between the reference frames will be performed on the mobile device to reconstruct the non-ROI (pixels) that are dropped on the server. Mean Opinion Score (MOS) measurements that are obtained from a panel of human will observes and evaluates the videos after the non-ROI are been reconstructed.

The remainder of this paper is organised as follows. Section 2 provides related work to spatial adaptation for slicing the video frames over a wireless network. Section 3 explains the proposed video streaming scenario. Section 4 adaptive the video according to the quantization parameters, while the analyses of the subjective data from the experiments study are presented in Section 5. Section 6 provides the experimental test results. Finally, we conclude this study in Section 7.

\section{RELATED WORK}

Several techniques have been proposed for video frames adaptation. Lai et al. [15] proposed a video encoding scheme based on visual attention model. The video frames are divided into several ROI with different attention levels. The attention model will provide the segmentation of the ROIs according to the saliency map. The visual attention scheme is propose to model the visual effects due to motion, and static attention. In static attention model, a saliency map is generated from each frame by the three channels saliency maps computation namely color contrasts, intensity contrasts, and orientation contrasts.

Liu et al. [16] proposed an ROI algorithm with adaptive background (non-ROI) skipping for scalable video coding. The ROI are encoded with high frames rate to ensure the smooth motion that can give a good visual quality. The algorithm will skip the non-ROI, as the non-ROI attracts less attention of viewer without decreases the quality of the video. The adaptive skipping decision depends on the motion activity of the non-ROI, while the bits are saved by skipping non-ROI, which are used to enhance the ROI spatial quality. The perceptual quality is significantly improved; since the human vision is tend to pay more attention to the ROI rather than the non-ROI.

Wang et al. [17] proposed a low-complexity content adaptive non-ROI skipping scheme for real-time video applications. The adaptive non-ROI skipping scheme will takes into account the frames contents variation in skip mode decision and rate control. The scheme will decides dynamically whether to skip the non-ROI macroblocks of the current frame and reallocate to the saved bits of the ROI. The scheme will allows skipping the non-ROI for every two consecutive frames and grouped them into a unit, where the non-ROI of the second frame is skipped (not coded but replaced by the macroblocks of the first frame in the same locations). The scheme will dynamically reallocate the saved bits due to the skipping to other regions, and adjusts the bit allocation for both frames and macroblock levels. The non-ROI will coded from the contents information of the current and previous frames.

The researchers identify the ROI as the most attractive object or region to the viewers. They considered the adaption methods to stream the selective regain (ROI) and to skip/drop the background (non-ROI), to cope with the bandwidth limitations.

\section{THE PROPOSED TECHNIQUE}

In this study, we propose a technique to reduce the video data by identifying and extracting the most motion regain in the video frames. The motion regain in the video frames are considered as Region Of Interest (ROI). The ROI with the reference frames are streamed to the mobile device. The mobile device will perform a reconstruction mechanism to the non-ROI pixels that are dropped on the server side based on the following steps:

\section{A. Identifying the ROI}

The streaming server will establish the connection according to the mobile request. The server will identify the position of the most motion regain (ROI) in the video frames and drop the pixels that are outside the ROI. The technique are used to identify the ROI is the Sum of Absolute Differences (SAD). The SAD is a commonly used technique for motion estimation in various video coding standards like H.264 [18]. The SAD value will be low except for the changes induced by the moving objects between frames. If there is a lot of motion within the frames, the SAD value will be relatively high, and if there is no motion then the SAD value will be less.

The SAD is computed to identify the estimated position of the ROI, as the consecutive video frames are scanned in two ways, vertically and horizontally:

- Vertically: Computing the SAD from left-to-right, is to identify the highest width (column) for the consecutive video frames, as shown in Fig. 1 and as in (1). The $\operatorname{SADv}(\mathrm{x})$, is calculated to identify the beginning and the ending of the highest difference value for each column in the video frame width.

$$
\operatorname{SAD}_{\mathrm{v}}(x)=\sum_{j=0}^{N-1} \sum_{z=1}^{L-1}\left|\mathrm{~F}_{\mathrm{z}}(x, \mathrm{j})-\mathrm{F}_{\mathrm{z}-1}(x, \mathrm{j})\right|
$$

- Horizontally: Computing the SAD from top-to-bottom, is to identify the highest height (row) for the consecutive video frames, as shown in Fig. 2 and as in (2). The $\mathrm{SADH}(\mathrm{y})$, is calculated to identify the beginning and the ending of the highest difference value for each row in the video frame height.

$$
\operatorname{SAD}_{\mathrm{H}}(y)=\sum_{i=0}^{M-1} \sum_{z=1}^{L-1}\left|\mathrm{~F}_{\mathrm{z}}(i, \mathrm{y})-\mathrm{F}_{\mathrm{z}-1}(i, \mathrm{y})\right|
$$

The average of the $\operatorname{SADv}(\mathrm{x})$ for the vertical scanning and $\mathrm{SADH}(\mathrm{y})$ for the horizontal scanning are calculated as in (3) and (4) respectively. 

video frames based on SADv $(x)$.

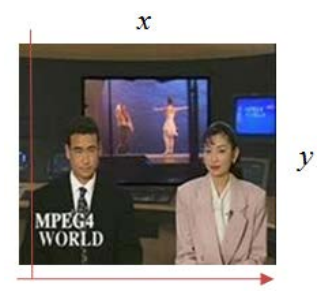

Figure 1. Scanning the consecutive

$$
\begin{aligned}
& \operatorname{Avg}(x)=\frac{1}{M} * \sum_{i=0}^{M-1} \operatorname{SAD}_{\mathrm{V}}\left(x_{i}\right) \\
& \operatorname{Avg}(y)=\frac{1}{N} * \sum_{j=0}^{N-1} \operatorname{SAD}_{\mathrm{H}}\left(y_{j}\right)
\end{aligned}
$$

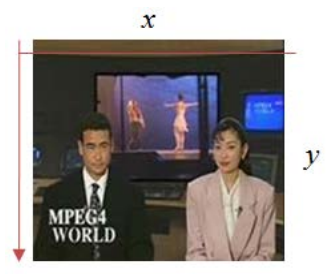

Figure 2. Scanning the consecutive video frames based on $\mathrm{SADH}(y)$.

Where $L$ is the length of the frame sequences, $N$ is the height and $M$ is the width.

The average values that are obtained from (3) and (4) will be used to identify the crossing point with the values that are obtained from (1) and (2). Where the crossing point represent the coordination points within the frame to identify the position and the resolution of the motion region, which it is the ROI in the video frames, as shown in Fig. 38.

The test videos are used in this work were the samples of video sequences Highway, Akiyo, Foreman, News, and Waterfall, with a resolution of 144 x 176 [19].

For Highway video, the $\mathrm{SADH}$ value is the largest when the ROI is close to the bottom of the frames as the highest differences among the intra-slices as shown in Fig. 4(b). The SADv value for the Highway video frames is very hard to estimate; therefore, it will be consider the complete width as shown in Fig. 4(a). The position and the resolution of the ROI for the Highway video can be estimate and as shown in

For Waterfall video, the SADv value is the highest in the middle of the frames but it is very hard to estimate the value of the $\mathrm{SADH}$, as the video is zooming out all the times, therefore it will be consider the complete height as shown in Fig. 3(b) and Fig. 5, respectively.

For Akiyo, and News videos, the SADH and SADv values are the highest in the middle of the frames as shown and the resolution is different, as the ROI for Akiyo has less motion than the News as shown in Fig. 3 (c) and Fig. 3 (e), respectively.

For Foreman video, the SADv value is the highest in the middle of the frames but it is very hard to estimate the value of the SADH, as the video is shaking all the times, therefore it will be considered the complete height as shown in Fig. 3(d) and Fig. 7, respectively.

The resolution of the ROI will be different from one video to another as each video had different characteristics, motion level and position as shown in Fig. 3. Fig. 3(a). in Fig. 6 and Fig. 8, respectively. While the ROI position
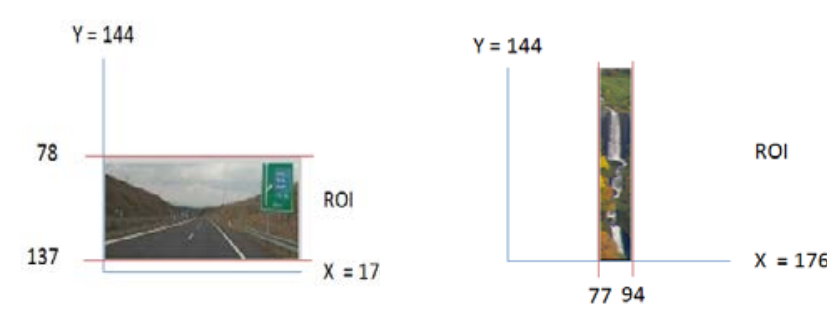

a. ROI for Highway.

b. ROI for Waterfall.
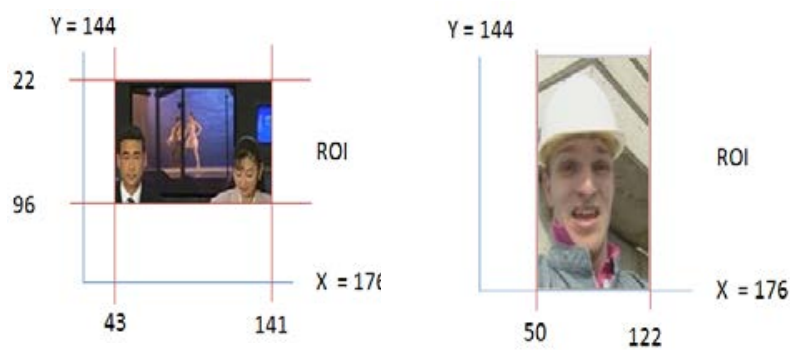

c. ROI for News.

d. ROI for Foreman.

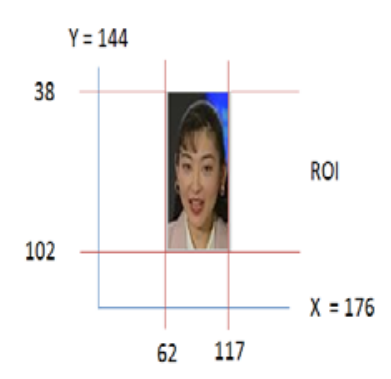

e. ROI for Akiyo
Figure 3. The ROI for the test videos.

\section{B. Extracting the ROI}

After the ROI it is been identify within the video frames, the server will split the video frames sequence into two parts. The first part is the reference frames, where the reference frames are set in this study is every fifth frame, as the maximum distance between frames that do not have high effect on the quality of the viewers' perception [20]. The second part is to extract the ROI from the frames between reference frames and drop the non-ROI pixels, as shown in Fig. 9(a). The reference frames with the ROIs are been encode by using H.264 and it will be transmit to the mobile device.

\section{Reconstructing the Video Frames}

The mobile device start receiving the video frames (reference frames and the ROIs). The frames are held in the buffer for reconstruction to the pixels that are outside the ROI. Linear interpolation [21] will be perform to reconstruct the dropped pixels (non-ROI) from the reference frames to return the frames to their original resolution, as shown in Fig. 9(b). The mobile device will play the video according to the playout rate. 


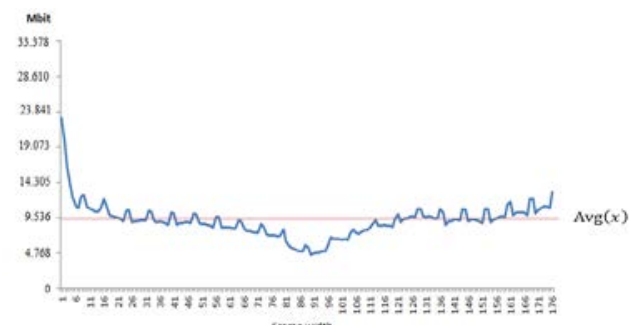

a. The $\operatorname{SADv}(x)$.

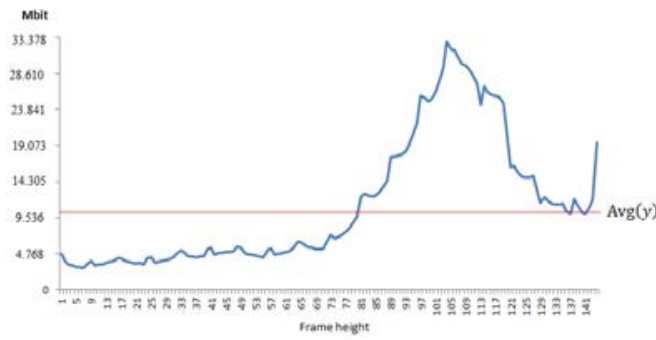

b. The $\operatorname{SADH}(y)$.

Figure 4. Highway video.

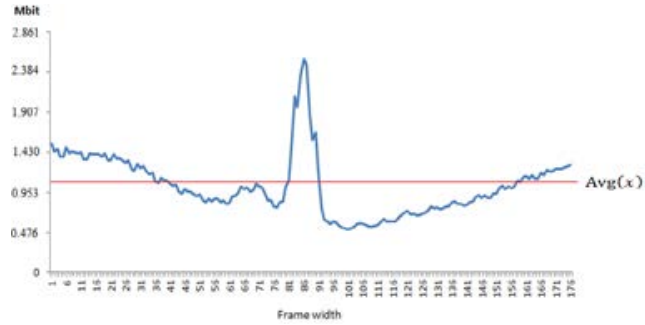

a. The $\operatorname{SADv}(x)$.

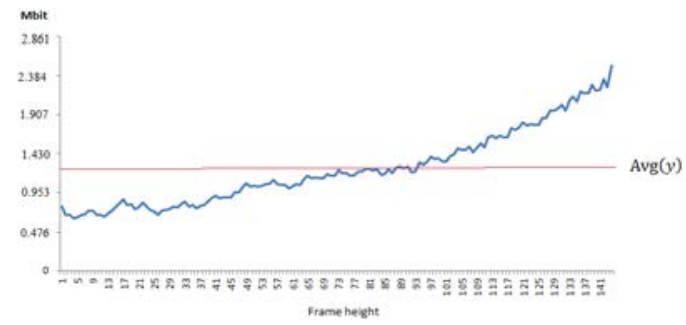

b. The SADH(y).

Figure 5. Waterfall video.

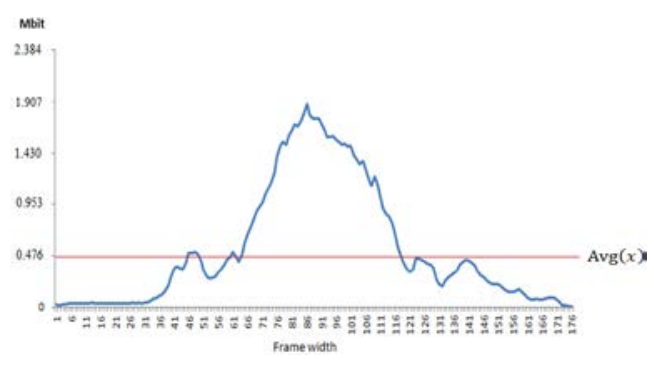

a. The $\operatorname{SADv}(x)$.

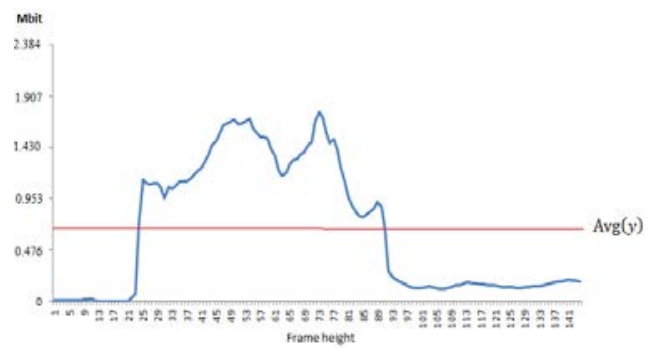

b. The $\operatorname{SADH}(y)$.

Figure 6. News video.

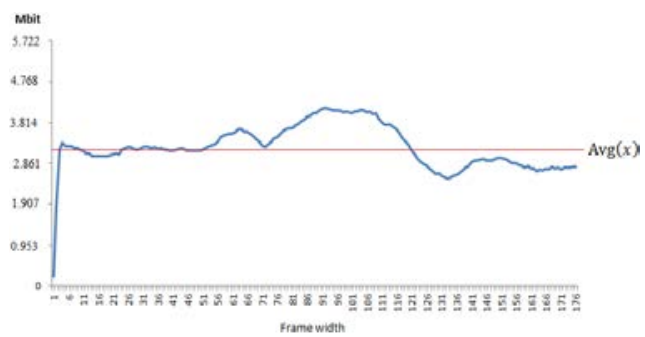

a. The $\operatorname{SADv}(x)$.

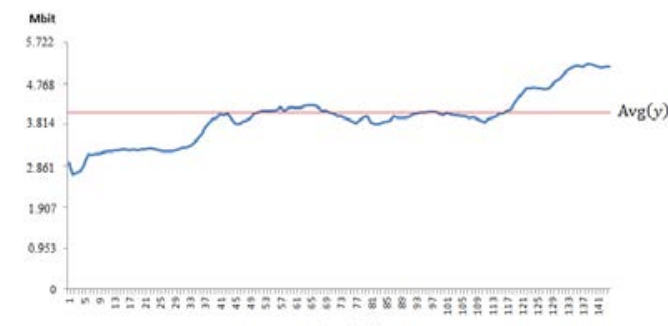

b. The $\mathrm{SADH}(y)$.

Figure 7. Foreman video.

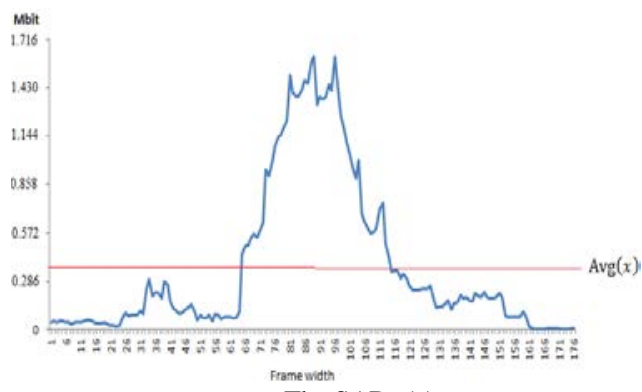

a. The $\operatorname{SADv}(x)$

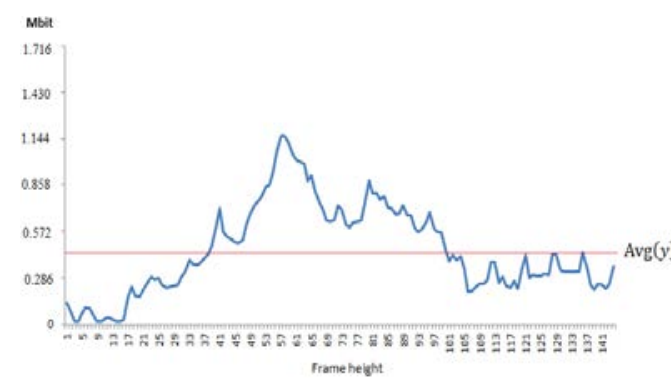

b. The $\operatorname{SADH}(y)$.

Figure 8. Akiyo video 


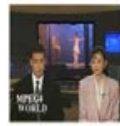

Frame No. 0 Reference Frame
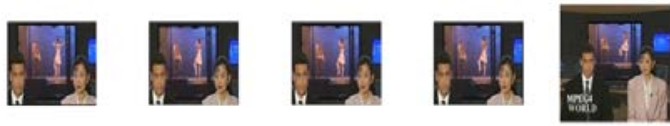

Frame No. 1

Frame No. 2

Frame No. 3

Frame No. 4

Frame No. 5

$\mathrm{RO}$

ROI

Reference Frame

a. Reference frames with ROI
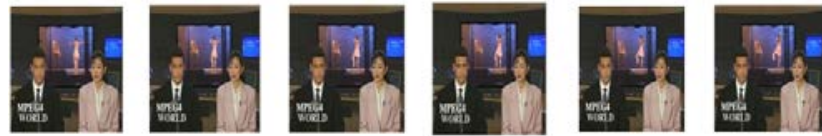

Frame No. 0

Frame No. 1

Frame No. 2

Frame No. 3

Frame No. 4

Frame No. 5

Refenence Frame Reconsitncted frame Reconstncted Frame Reconstruxted frame Reconstructed Frame Reference Frame

b. The reconstructed frames.

Figure 9. The proposed scenario.

\section{QUANTIZATION PARAMETER ADAPTATION}

The video will encode to obtain the optimum visual quality to the available network bandwidth. The bit allocation for the video should achieve the tradeoff between encoding the video quality and bandwidth limitation.

The bit allocation for the video is encoded by using H.264 ffmpeg codec [22]. The videos are encoded to identify the effectiveness of the bit rates and the quantization parameter (QP) on the encoding size.

Two scenarios are proposed to encode the videos, the first scenario; where the original videos are encoded with default QP for a bit rate of $64 \mathrm{kbps}$. The second scenario (the proposed scenario) where the videos are encoded with a bit rate of $128 \mathrm{kbps}$, as shown in Table 1 . The encoding size are different from one video to another, as the videos had different characteristics. Encoding the videos in the second scenario with adaptive QP is to gain equivalent encoding size for the videos that are in the first scenario that can cope with the bandwidth limitation.

\section{SUBJECTIVE VIEWING TEST}

\section{A. Test Methods}

It is well known that the peak signal-to-noise ratio (PSNR) does not always rank quality of an image or video sequence in the same way as a human being. There are many other factors considered by the human visual system and the brain [23]. One of the most reliable ways of assessing the quality of a video is subjective evaluation of the Mean Opinion Score (MOS). MOS is a subjective quality metric obtained from a panel of human observers. It has been regarded for many years as the most reliable form of quality measurement technique [24].

\section{B. Testing Materials and Environments}

The videos are displayed on a 17 inch FlexScan S2201W LCD computer display monitor of type EIZO with a native resolution of $1680 \times 1050$ pixels.
TABLE I. THE SIZE OF THE ENCODING VIDEOS FOR THE TWO SCENARIOS.

\begin{tabular}{|l|c|c|c|c|c|c|c|}
\hline $\begin{array}{c}\text { Test } \\
\text { Videos }\end{array}$ & $\begin{array}{c}\text { Size } \\
(\mathrm{KB}), \\
\text { QP=2 }\end{array}$ & $\begin{array}{c}\text { Scenario 1: } \\
\text { QP }\end{array}$ & $\begin{array}{c}\text { Size } \\
(\mathrm{KB})\end{array}$ & $\begin{array}{c}\text { Coding } \\
\text { Efficiency } \\
\text { Gain }\end{array}$ & $\begin{array}{c}\text { Scenario 2: } \\
\text { QP }\end{array}$ & $\begin{array}{c}\text { Size } \\
(\mathrm{KB})\end{array}$ & $\begin{array}{c}\text { Coding } \\
\text { Efficiency } \\
\text { Gain }\end{array}$ \\
\hline Highway & 4564 & 14 & 707 & $\mathbf{8 4 , 5 0}$ & 10 & 640 & $\mathbf{8 5 , 9 7}$ \\
\hline Waterfall & 1280 & 10 & 165 & $\mathbf{8 7 , 1 0}$ & 3 & 138 & $\mathbf{8 9 , 2 1}$ \\
\hline News & 1128 & 11 & 167 & $\mathbf{8 5 , 1 9}$ & 6 & 179 & $\mathbf{8 4 , 1 3}$ \\
\hline Foreman & 616 & 12 & 146 & $\mathbf{7 6 , 2 9}$ & 11 & 144 & $\mathbf{7 6 , 6 2}$ \\
\hline Akiyo & 350 & 6 & 165 & $\mathbf{5 2 , 8 5}$ & 3 & 169 & $\mathbf{5 1 , 7 1}$ \\
\hline
\end{tabular}

The videos are displayed with resolution of $144 \times 176$ pixels in the centre of the screen with a black background with a duration of 66 seconds for Highway video and 10 seconds for Akiyo, Foreman, News and Waterfall videos.

The MOS measurements are used in this study to evaluate the video quality according to the guidelines outlined in the BT.500-11 recommendation of the radio communication sector of the International Telecommunication Union (ITU-R). We use a lab with controlled lighting and set-up according to the ITU-R recommendation. The score grades in this methods range from 0 to 100 . These ratings are mapped to a 5-grade discrete category scale labelled with Excellent, Good, Fair, Poor and Bad [25].

The subjective experiment was conducted at Blekinge Institute of Technology in Sweden. The participant of thirty non-expert test subjects, 27 males and 3 females. They were all university students and their ages range from 20 to 35 .

The users observed two scenarios for displaying the videos; the first scenario is to display the video for a low bit rate for the original video and the second scenario by implementing our proposed technique with a high bit rate encoding, where the playing rates for both scenarios is 30 frames per second.

The amount of data gathered from the subjective experiments with respect to the opinion scores that were given by the individual viewers. Concise representation of this data is achieved by calculating the conventional statistics such as the mean score and 95\% confidence interval.

\section{EXPERIMENTAL RESULTS}

A panel of users evaluates the two scenarios according to the Mean Opinion Score (MOS) measurements. In the first scenario, the original videos are decoded with a bit rate of $64 \mathrm{kbps}$. The second scenario (the proposed scenario), the video are decoded with a bit rate of $128 \mathrm{kbps}$ with linear interpolation are performed to the second scenario to reconstruct the pixels that are outside the ROI, as shown in Fig 10.

For Highway videos the observers evaluates the both scenarios almost within the same score range, as an indicator that the observers had similar opinion to the quality of the videos. 


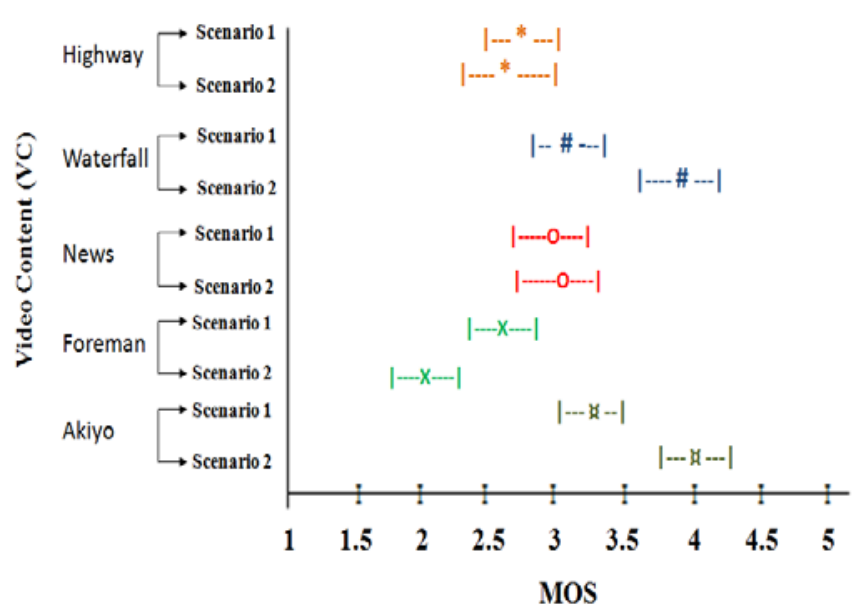

Figure 10. The MOS for different videos content.

For Waterfall videos, the MOS for the first scenario it is less than 3.5, while for the second scenario is more than 3.5, as an indicator that the viewers give high score to our proposed scenario.

For News videos, both scenarios almost within the same score range, but the second scenario is slightly higher than the first scenario.

For Foreman videos, the MOS for the second scenario is the lowest score than the first scenario, as the observers manage to recognize the effect of the interpolation on the video frames. The reason for that is, Foremen video is shaking all the time and it is hard to reconstruct the dropped pixels outside the ROI, although the first scenario is encoded with a low bit rate.

For Akiyo videos, the MOS for the first scenario is less than 3.5, while the second scenario is more than 3.5, as an indicator that, the viewers give high score to our proposed scenario.

\section{CONCLUSION}

In this study, we proposed an adaptive scheme to reduce the amount of video data that are streamed to the mobile device over wireless networks. The adaption scheme is based on computing the Sum of Absolute Differences (SAD) to identify, and extract the ROI. The ROI resolution and the encoding size are different from one video to another as each video had different motion level, which falls in different position within the video frames.

The receiving video stream on the mobile device, will reconstruct the dropping pixels that are outside the ROI by performing linear interpolation and from the reference frames.

It seems that the higher $\mathrm{SAD}$ values results as an indicator to the motion and important region within the video frames. The quality of the video for the second scenario (the proposed scenario) is higher than the first scenario, where both scenarios had approximately equivalent encoding size.
A panel of users observes and evaluates the two scenarios by using the MOS measurements. It shows that, the scores are different from one video to another as the resolution and the position of the ROI is different from one video to another. It been notice from that, the MOS is high for the videos like Waterfall, Akiyo and News, while for a video like Foreman, the MOS it was showing the lowest score as the video frames are shaking all the times as the reconstruction to the pixels that are outside the ROI been notice very clearly by the viewers.

Even the quality for some videos is degraded; but it could still be a satisfactory technique to reduce the encoding size for the videos that are streamed over limited bandwidth.

\section{ACKNOWLEDGMENT}

We would like to thank the students at Blekinge Institute of Technology, Sweden for participating in the subjective experiments. We also would like to thank the Swedish Knowledge Foundation for sponsoring a part of this work through the project QoEMoS (d-nr 2008/0520).

\section{REFERENCES}

[1] D. Tian, X. Li, G. Al-Regib, Y. Altunbasak, and J. R. Jackson,“ Optimal Packet Scheduling for Wireless Video Streaming with Error-Prone Feedback," Proc. IEEE Wireless Communications and Networking Conference (WCNC 04), March 2004, pp. 1287-1292, doi: 10.1109/WCNC.2004.1311374.

[2] J-Y. Chang and H-L. Chen, “ Dynamic-Grouping Bandwidth Reservation Scheme for Multimedia Wireless Networks," IEEE Journal on Selected area in Communications, vol. 21, Dec. 2003, pp. 1566-1574, doi: 10.1109/JSAC.2003.814863.

[3] G-R. Kwon, S-H. Park, J-W. Kim, and S-J. Ko, “ Real-Time R-D Optimized Frame-Skipping Transcoder for Low Bit Rate Video Transmission,” Proc. IEEE $6^{\text {th }}$ International Conference on Computer and Information Technology (CIT 06), Sept. 2006, doi : 10.1109/CIT.2006.158.

[4] H. Luo, M-L. Shyu, and S-C. Chen,“ An End-to-End Video Transmission Framework with Efficient Bandwidth Utilization,” Proc. IEEE International Conference on Multimedia and Expo. (ICME 04), June 2004, pp. 623-626, doi: 10.1109/ICME.2004.1394269.

[5] F. Yang, Q. Zhang, W. Zhu, and Y-Q. Zhang, “ Bit Allocation for Scalable Video Streaming over Mobile Wireless Internet," Proc. IEEE $23^{\text {rd }}$ Computer and Communications Societies, March 2004, pp. 2142-2151, doi: 10.1109/INFCOM.2004.1354621.

[6] J.-H. Lee and C. Yoo, " Scalable ROI Algorithm for H.264/SVC-Based Video Streaming," IEEE Transactions on Consumer Electronics, vol. 57, May 2011, pp. 882-887, doi: 10.1109/TCE.2011.5955236.

[7] H. Liu, W. Zhang, S. Yu, and X. Yang, “ Channel-Aware Frame Dropping for Cellular Video Streaming,” Proc. IEEE International Conference on Acoust., Speech, Signal Processing (ICASSP 06), May 2006, pp. 409- 412, doi: 10.1109/ICASSP.2006.1661299.

[8] D. Grois, E. Kaminsky, and O. Hadar, “ ROI Adaptive Scalable Video Coding for Limited Bandwidth Wireless Networks,” Proc. IFIP Wireless Day (WD 10), Oct. 2010, pp. 1-5, doi: 10.1109/WD.2010.5657709.

[9] L. Al-Jobouri, M. Fleury, S. S. Al-Majeed, and M. Ghanbari, " Effective Video Transport over WiMAX with Data Partitioning and Rateless Coding," Proc. IEEE $10^{\text {th }}$ International Conference on Computer and Information Technology (CIT 10), July 2010, pp. 767 - 772, doi: 10.1109/CIT.2010.147. 
[10] S. Gong, J. Yang, and S. Zhang, “ A Novel Content-Based Video Coding Scheme for Robust Video Transmission over Ad Hoc Networks," Proc. IEEE 5th International Conference on Wireless Communications, Networking and Mobile Computing (WiCom 09), Sept. 2009, pp.1-5 ,doi: 10.1109/WICOM.2009.5302389.

[11] J-H. Lee and C. Yoo, " Scalable ROI Algorithm for H.264/SVC-Based Video Streaming," IEEE Transactions on Consumer Electronics, vol. 57, May 2011, pp. 882-887, doi: 10.1109/TCE.2011.5955236.

[12] M. Inoue, H. Kimata, K. Fukazawa, and N. Matsuura, "Partial Delivery Method with Multi-Bitrates and Resolutions for Interactive Panoramic Video Streaming System,” Proc. IEEE International Conference on Consumer Electronics (ICCE 11), January 2011, pp. 891-892,doi: 10.1109/ICCE.2011.5722922.

[13] L. Czuni, G. Csaszar, and A. Licsar, " Estimating the Optimal Quantization Parameter in H.264," Proc. IEEE $18^{\text {th }}$ International Conference on Pattern Recognition (ICPR 06), August 2006, pp. 330-333, doi: 10.1109/ICPR.2006.502.

[14] M. Hrarti, H. Saadane, M. Larabi, A. Tamtaoui, and D. Aboutajdine, “ Adaptive Quantization Based on Saliency Map at Frame Level of H.264/AVC Rate Control Scheme,” Proc. IEEE $3^{\text {rd }}$ European Workshop on Visual Information Processing (EUVIP 11), July 2011 ,pp. 61- 66, doi: 10.1109/EuVIP.2011.6045539.

[15] W. Lai, X-D. Gu, R-H. Wang, W-Y. Ma, and H-J. Zhang, "A Content Based Bit Allocation Model for Video Streaming," Proc. IEEE International Conference on Multimedia and Expo (ICME 04), June 2004 , pp. 1315 - 1318, doi: 10.1109/ ICME.2004.1394469.

[16] Q. Liu, R-M. Hu, and Z. Han,“Adaptive Background Skipping Algorithm for Region-of-Interest Scalable Video Coding," Proc. IEEE $11^{\text {th }}$ Singapore International Conference on Communication Systems (ICCS 08), November 2008, pp. 788 - 792, doi: 10.1109/ICCS.2008.4737292.
[17] H. Wang, Y. Liang, and K. El-Maleh, “ Real-Time Region-ofInterest Video Coding using Content-Adaptive Background Skipping with Dynamic Bit Reallocation,” Proc. IEEE International Conference on Acoustics, Speech and Signal Processing ( ICASSP 06), May 2006, pp. 45-48, doi: 10.1109/ ICASSP.2006.1660275.

[18] A. Dimou, O. Nemethova, and M. Rupp, " Scene Change Detection for H.264 Using Dynamic Threshold Techniques," Proc. $5^{\text {th }}$ EURASIP Conference on Speech and Image Processing, Multimedia Communications and Service, July 2005.

[19] trace.eas.asu.edu/yuv/index.html.

[20] A. Kaur, P. Sircar, and A. Banerjee, “ Interpolation of Lost Frames of a Video Stream Using Object Based Motion Estimation and Compensation,” Proc. IEEE India Conference (INDICON 08), Dec. 2008, pp. 40-45, doi: 10.1109/ INDCON.2008.4768798.

[21] Y.-C. Peng, H.-A. Chang, C.-K. L. Chen, and H. C.-J. Kao, "Integration of Image Stabilizer with Video Codec for Digital Video Cameras," Proc. IEEE Inter. Symp. Circuits and Systems (ISCAS 05), May 2005, pp. 4871- 4874, doi: 10.1109/ISCAS.2005.1465724.

[22] www.ffmpeg.org.

[23] M. Martinez-Rach, O. López, P. Piñol, M.P. Malumbres, J. Oliver, and C.T. Calafate, " Quality Assessment Metrics vs. PSNR under Packet Loss Scenarios in MANET Wireless Networks," Proc. International Workshop on Mobile Video, June 2007, pp. 31-36, doi:10.1145/1290050.1290058.

[24] M. Martinez-Rach, O. López, P. Piñol, M.P. Malumbres, J. Oliver, and C.T. Calafate, " Behavior of Quality Assessment Metrics under Packet Losses on Wireless Networks,” XIX Jornadas de Paralelismo, Castellón, September 2008.

[25] International Telecommunication Union. Methodology for the Subjective Assessment of the Quality of Television Pictures. ITU-R, Rec. BT.500-11, 2002. 